\title{
Analysis of Korea-Massive Open Online Courses in medical and dental education
}

\author{
Jieun Lee ${ }^{1}$, Sun-Kyoung Yu ${ }^{1,2 *}$ \\ ${ }^{1}$ Department of Education, College of Education, Chosun University, Gwangju, Korea \\ ${ }^{2}$ Department of Oral Anatomy, College of Dentistry, Chosun University, Gwangju, Korea
}

The objectives of this study were to analyze the status and the instructional design strategies of medical and dental courses in KoreaMassive Open Online Course (K-MOOC), and to present a practical direction for application in future dental education. Among 30 courses categorized as medical education in K-MOOC as of December 2018, only three cases were selected for this study. The courses were analyzed based on the proposed instructional design strategies for facilitating online interaction among participants-instructors, teaching assistants, and learners. In addition, this study reviewed the current status of free online dentistry courses in MOOC including K-MOOC. The number of basic medical science courses was five in total, however, the dentistry courses in K-MOOC was not offered yet. Regarding the format, the medical courses teaching format was similar to a lecture-based traditional classroom, which was characterized with one-way content delivery style. However, the current courses were appropriately designed with the proposed instructional design strategies to facilitate online interaction. In addition, MOOC platforms had 11 dentistry courses in 2018, even though most of them started to operate the courses recently. The results of this study denote current scarcity of dental MOOCs that could have educational potential benefits from well-structured courses with rich media delivered online and encourage dental educators to consider MOOCs as alternative educational opportunities. In addition, it provides the instructional design strategies that should be considered in designing new MOOCs. It also provides a practical direction for the development of personalized learning dentistry courses in K-MOOC.

Key Words: Dental education, Electronic learning, Instructional design strategies, Korea-Massive Open Online Course

(c) This is an open-access article distributed under the terms of the Creative Commons Attribution Non-Commercial License (http://creativecommons.org/licenses/by-nc/4.0) which permits unrestricted noncommercial use, distribution, and reproduction in any medium, provided the original work is properly cited.

\section{서 론}

정보통신기술의 급격한 발전은 교실 수업 중심의 전통적 대 학교육의 패러다임으로부터 네트워크와 인터넷 환경을 기반 으로 한 고등교육으로의 변화를 촉진하였다[1]. 최근 전 세계 각 학문 분야에서 급격한 성장을 보이는 대형 온라인 공개강좌 (Massive Open Online Courses, MOOC)는 2008년 캐나다 마
니토바 대학교에서 처음 사용된 용어로, 수강인원에 제한 없이 (massive), 모든 사람이 수강 가능하며(open), 웹 기반으로(online) 미리 정의된 학습목표를 위해 설계된 강좌(course)로 정의 된다[2,3]. MOOC는 기존의 Open Course Ware에서 제공했던 일방향적 강의 콘텐츠의 무료 공개를 넘어 온라인 수업의 장점 을 강화하고 오프라인 수업에서 기대할 수 있는 학습자와 교수 및 다른 학습자 간의 양방향의 상호작용이 가능한 새로운 교육

Received January 30, 2019; Revised February 12, 2019; Accepted February 18, 2019

Corresponding author: Sun-Kyoung Yu, Department of Oral Anatomy, College of Dentistry, Chosun University, 309 Pilmun-daero, Dong-gu, Gwangju 61452, Korea.

Tel: +82-62-230-6357, Fax: +82-62-224-3706, E-mail: sky@chosun.ac.kr

Copyright @ 2019, Oral Biology Research Institute 
환경을 제시한다[2].

2010년대 북미를 중심으로 시작된 MOOC는 이러한 온라인 기반 상호작용 학습 환경이라는 특징 및 고등교육 기회의 무료 제공이라는 지향 가치로 인하여 학습자 및 교수자의 큰 관심과 기대감을 불러일으켰다. 최근 세계 여러 국가에서 고등교육 콘 텐츠의 공개와 공유를 지향하며 다양한 분야에서 $\mathrm{MOOC}$ 를 개 발하여 운영하고 있다[4,5]. 이와 같은 세계적인 교육 흐름에 따 라 우리나라도 국가평생교육진흥원(옛 국가평생교육원)의 주도 하에 2015년 10월에 Korea-MOOC (한국형 온라인 공개강좌, $\mathrm{K}-\mathrm{MOOC}$ )사업이 시작되었다. 최초로 서울대학교와 KAIST 등 10 개의 국내 대학을 시범사업 대학으로 지정하여 27개 MOOC 강좌 서비스를 개시하였다[3,5]. 시작 2개월 만에 이들 강좌에 4 만 3000여 명이 수강 신청을 하여 우리나라에서도 MOOC에 대 한 높은 수요와 관심이 있다는 것이 드러나게 되었다[5,6].

우리나라가 K-MOOC 서비스를 개시하기 전인 2013년 12월 해외 $\mathrm{MOOC}$ 사이트에서는 건강과 의학에 관련된 225 개의 강 좌가 개설되었으며, 수강 및 청강 가능한 강좌 수도 무려 98개 나 되었다[7]. 이러한 MOOC의 교육적 가능성 및 높은 수요, 양 질의 다양한 강좌의 제공 가능성 및 대학교육 패러다임의 변화 요구로 인해 치의학 교육 분야도 MOOC의 활용에 대해 적극적 으로 논의하기 시작하였다[8-10]. 한국치의학교육평가원에서 는 치의학 교육에 있어 다양한 임상환경에 따른 의사결정과 환 자에게 시행하는 기본적 기술 적용을 촉진하기 위해 학습자 특 성을 고려한 문제바탕학습(problem-based learning, PBL)이나 플립 러닝(flipped learning)과 같은 향상된 방식의 학습모형이 적용된 교육방식을 요구하고 있다.

학습은 학습자가 교수자, 동료학습자와 정보와 의견을 활발 히 교환하며 의미를 만들어가는 양방향적 자기주도적 의사소 통인 상호작용을 통해 촉진된다[11,12]. 높은 수준의 상호작용 은 학습자의 성공적인 학습에 기여하며, 이는 강의실 수업뿐 아니라 이러닝(e-learning)에서도 역시 중요하다[13]. Moore [14]는 온라인 강좌에서 이루어지는 상호작용을 3가지 유형으 로 정리하여 제시하였다. 첫째, 학습자와 콘텐츠 간의 상호작용 (learner-content interaction)으로 학습자가 텍스트, 교육 영 상, 오디오 영상 등의 매체를 통해 자기주도적으로 학습하는 과 정이다[15,16]. 둘째, 학습자와 교수자 간 상호작용(learnerinstructor interaction)을 통하여 학습자의 학습동기가 발현되 고 흥미를 지속시켜 학습자 스스로의 학습을 유도한다[15]. 셋 째, 학습자와 학습자 간의 상호작용(learner-learner interaction)을 통하여 학습자는 온라인 학습 환경에서 자신의 존재감 을 확인하며 소속감을 느끼게 되고, 동료 학습자에 대한 비교 관 찰을 통해 자신의 학습에 대한 성찰이 촉진될 수 있다[15]. 종합 해보면, $\mathrm{MOOC}$ 의 교수 설계에서 이러한 학습자와 콘텐츠, 교
수자, 학습자 간의 상호작용 설계 전략의 적용은 학습자들의 학 업성취 및 참여와 수강 완료 비율을 높이는데 기여할 수 있다 $[11,12,13,15,17]$.

본 연구에서는 $\mathrm{K}-\mathrm{MOOC}$ 에서 이루어지고 있는 치의학 분야 의 강좌 실태 및 설계 전략을 분석하기 위하여 샘플 강좌를 선정 해야 했다. 이를 위해 먼저 2018년 12월에 K-MOOC가 제공하 고 있는 강좌목록을 살펴보았다. K-MOOC의 전체 489 개 강좌 는 의약, 인문, 사회, 교육, 공학, 자연, 예체능의 7 개로 대분류되 어 있었는데, 이 중 의약 분야는 2017년에 2개, 2018년에 28개 가 개설되어 총 30 개 강좌로 약 $6 \%$ 에 해당하는 아주 적은 실정 이었다. 이들 강좌를 다시 중분류로 나누면 의료 18 개, 간호 2 개, 치료·보건에 10 개 강좌였고, 치의학에 관련한 강좌는 전혀 없었다.

따라서 본 연구에서는 의료 상식적인 교양 성격이거나 다루 는 학문의 영역이 다른 임상 의학 강좌를 제외하고, 학문 영역이 유사하여 치의학 교육에 적용할 수 있는 기초 의학 분야의 세 강 좌를 분석 대상 강좌로 선정하였다. 선정한 기초 의학 강좌의 요 소와 상호작용 설계 세부 전략을 분석하여, 향후 $\mathrm{K}-\mathrm{MOOC}$ 에서 치의학 분야 강좌 개설에 적용할 수 있는 시사점을 도출하고자 하였다. 또한 면대면 교실에서 교수자의 강의 위주로 이루어지 던 치의학 교육에 새로운 학습 환경에 대한 대안을 학문분야에 소개하고, 향후 치의학 교육에서 $\mathrm{K}-\mathrm{MOOC}$ 의 적용을 위한 실제 적 방향성을 모색하고자 하였다.

\section{이론적 배경}

\section{$\mathrm{MOOC}$ 의 현황}

MOOC는 원격교육(distance education)과 온라인교육(online learning), 개방교육자료(open educational resources) 운 동에 뿌리를 두고 있다[18]. 2011년 스탠퍼드 대학교에서 컴퓨 터공학 분야 3 개의 강좌를 온라인을 통해 전 세계에 개방하면 서 세계의 이목이 집중되었다[2]. 이후 미국에서 $\mathrm{MOOC}$ 최초의 플랫폼인 Udacity가 개설되었고, 이후, 교육에서의 평등, 개방, 공유, 전문성 등의 기치를 걸고 Coursera, edX 등 MOOC 플랫 폼이 앞 다투어 개설되어, 폭발적인 인기와 함께 유럽과 아시 아 지역으로 급속하게 확산되었다[5,15,19]. 이러한 배경으로는 $\mathrm{MOOC}$ 가 학습자가 수동적으로 듣는 기존의 온라인 학습 콘텐 츠와 달리 학습자와 교수자 및 다른 학습자 간 질의응답, 토론, 과제 제출 등과 같은 양방향 상호작용이 이루어지는 질 높은 학 습 환경에서 무료로 학습할 수 있기 때문이다[3,5].

그러나 이러한 세계적인 관심과 급속한 양적 성장에 비해 $\mathrm{MOOC}$ 를 통하여 수강 신청한 강의수에 비하여 성공적으로 학 습을 완료한 숫자는 전체적으로 $4 \%-5 \%$ 에 불과하다. MOOC 
를 통해 고등교육 기회에 대한 접근성이 증대되었지만, 실질적 인 학습 효과 면에서는 아직 극히 미약하다[18]. 그 결과 MOOC 를 좀 더 체계적이고 효과적으로 개발, 운영하기 위해 다양한 연 구가 이루어지고 있으며, $\mathrm{MOOC}$ 의 사이즈와 형태도 다양해지 고 있다[18]. 또한 학습자의 다양하고 개별화된 요구 및 문화·사 회적 특성을 고려한 $\mathrm{MOOC}$ 의 설계에 대한 요구가 대두되었고, 국가별로 상황에 적합한 접근방식의 차별화가 이루어지고 있을 뿐만 아니라 최신 테크놀로지를 적용하여 MOOC의 형태가 발 전, 변화하고 있다[5,18].

\section{Korea-Massive Open Online Courses}

$\mathrm{K}-\mathrm{MOOC}$ 는 대학 교육의 실질적인 기회 균형을 실현하고 평 생학습 기반을 마련하여 국가 인적자원 개발에 기여할 것을 목 표로 교육부와 사업 주관기관인 국가평생교육진흥원과 한국교 육학술정보원, 한국대학교육협의회, 한국교육방송공사(EBS) 등 관계기관 및 참여 대학 간의 긴밀한 협력을 통해 정부 주도하에 추진되고 있다[3,20]. 향후 국제적 호환성과 사용자 편의성 등 을 고려하여 MIT와 Harvard에서 개발한 “Open edX 플랫폼" 을 활용하여 2015년 10월부터 K-MOOC 플랫폼(http://www. kmooc.kr)을 통해 서비스를 개시하였다. 2018년 12월 기준으 로 500여 개의 강좌를 제공 및 운영하고 있다[20].

\section{미래의 $\mathrm{MOOC}$ 방향과 보건 의료 분야}

온라인 테크놀로지 기반의 $\mathrm{MOOC}$ 가 교육적 기대 효과를 얻 기 위해서는 다양한 특성을 가진 학습자의 수준과 필요에 맞 게 설계, 개발할 필요가 있다. 이러한 목적으로 교육학 분야에 서 이루어진 여러 선행 연구의 결과를 종합하여 Jung [18]은 3 가지의 $\mathrm{MOOC}$ 모형을 제시하였다. 첫째, 상황적 무크(situated $\mathrm{MOOC})$ 는 PBL과 유사한 형태로 실제 상황에서 일어난 사례 기 반의 온라인 학습 활동 형태를 적용한 수업이다. 둘째, 시각적 무크(visual MOOC)는 콘텐츠 및 환경을 멀티미디어를 활용하 여 시각화하려는 것으로 풍부하고 효율적인 네트워크와 인터넷 환경을 가지는 한국인 학습자에게 효과적인 전략이다. 마지막, 투크(targeted open online courses, TOOC)라는 유형은 대형 강좌인 $\mathrm{MOOC}$ 의 한계점을 극복하고 특정 학습자 집단의 필요 와 요구에 맞는 강좌 설계와 운영을 위한 강좌이다. 따라서 고급 기능을 가진 유료 $\mathrm{MOOC}$ 강좌가 점점 늘어나는 현 추세에 맞춰 향후 많은 $\mathrm{MOOC}$ 가 특정 집단을 목표로 하여 개발되는 TOOC 형태로 전환될 것으로 예상하고 있다[5,18].

보건 의료 분야에서 $\mathrm{MOOC}$ 에 대한 반응은 의료 윤리, 학습 콘텐츠의 질적 부분, 테크놀로지의 기술적 부분 등으로 인하여 일관적이지 않지만[7,21,22], 대체적으로 공공 의료와 의학 교 육에 있어 긍정적인 교육적 효과를 기대하고 있다[7,23]. Johns
Hopkins 대학은 2012년에서 2013년에 걸쳐 다수의 공공 의 료 분야의 MOOC 강좌를 제공하였는데[22], 이를 통하여 의료 불균등을 해소할 수 있고 주요 건강 주제에 관한 공공보건 교육 의 가능성 및 의사와의 언어적 간격 극복으로 전문적 치료 가 능성이 높아질 것으로 기대하고 있다[7]. 또한 평생교육 측면에 서 의료인은 매년 의무적으로 보수교육을 받아야 하는데, 이 역 시 $\mathrm{MOOC}$ 를 활용한다면 그 편리성과 경제적 장점을 기대할 수 있을 것이다[24]. 마지막으로 의과대학의 교육에서는 플립 러 닝 형태와 유사하게 학생은 수업 전 $\mathrm{MOOC}$ 를 통하여 자율 학 습을 한 후, 실제 강의실에서는 교수자 및 다른 학생들과 더 심 도 있는 토론이나 학습활동으로 정교화된 상호작용을 할 수 있 을 것이고, 그 결과 더 높은 학습결과를 기대할 수 있을 것이다 $[7,18,25,26]$.

이처럼 $\mathrm{MOOC}$ 의 발전적이고 전문적인 변화와 보건 의료 분 야에서의 긍정적 시각은 치의학 교육에도 적극적으로 반영될 것이다. 따라서 향후 $\mathrm{K}-\mathrm{MOOC}$ 플랫폼에 제공할 치의학 교육 $\mathrm{MOOC}$ 의 설계와 개발을 위한 실천적 방향성을 모색할 필요가 있겠다.

\section{대상 및 방법}

\section{연구 설계와 연구 대상}

본 연구는 치의학 교육에서 $\mathrm{MOOC}$ 개발을 위한 실제적 방 향성을 모색하기 위하여 K-MOOC에서 이루어지고 있는 의학 분야의 강좌 및 해외 $\mathrm{MOOC}$ 에서 개설되어 있는 치의학 강좌 의 실태와 설계 전략을 분석하였다. 2018년 12월 기준으로 K$\mathrm{MOOC}$ 에 제공되어 있는 의약 분야 MOOC는 총 30 개 강좌였 으나 치의학 강좌는 한 강좌도 개설되어 있지 않았다. 이에 의료 상식적인 교양 성격이거나 다루는 학문의 영역이 다른 임상 의 학 강좌를 제외하고, 학문 영역이 같아 치의학 교육에 적용할 수 있는 기초 의학 강좌를 연구대상으로 선별하였다. 선별된 기초 의학 강좌는 중분류가 의료 4 개와 치료·보건 1 개로 총 5 개의 강 좌가 개설되어 있었다. 5 개 강좌 중 치료·보건 강좌의 경우 $\mathrm{K}-$ $\mathrm{MOOC}$ 상의 중분류가 잘못되어 있었지만 다루는 학문 영역을 파악한 결과 부합하여 연구 대상에 포함하였다.

강좌는 수강 기간 및 종료 여부에 따라 current (ongoing), archived, upcoming, self-paced로 분류되어 있고[15], 선별된 5 개의 강좌 중 연구시점인 2018년 12월에 수강 및 청강이 가능 한 강좌인 세 강좌를 최종 선정하였다. 먼저 의료분야에서 "의 료인을 위한 현대적 리더쉽”은 보건의료인을 대상으로 병원경 영에 관련한 수업을 하는 강좌로 2018년 4월 2일부터 2018년 7월 22일까지로 이미 종료되어 청강이 불가능하여 제외하였다. 


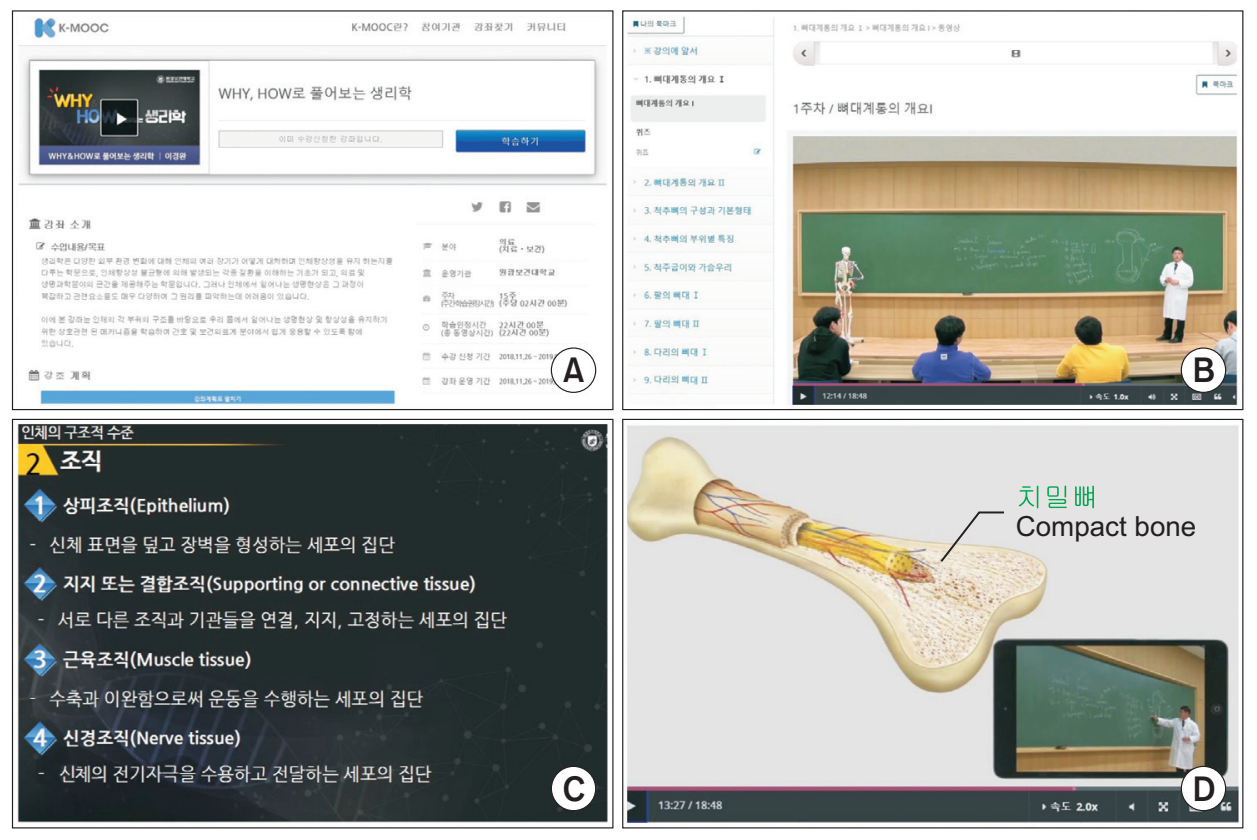

Fig. 1. Screenshots taken to show common features of basic medical science courses in Korea-Massive Open Online Courses. Course introduction (A), long teaching times of one-person instructor (B), learning materials (C), advanced materials using computer graphics (D).
두 번째 “보이지 않는 미생물 세계”는 일반인과 보건의료인을 대상으로 미생물의 특징과 항생제 등 관련 주제를 학습 목표로 하며, 2018년 10월 8일부터 2018년 12월 16일까지 운영하고 있었으나 등록이 마감되어 수강이 불가능하여 역시 제외하였 다. 세 번째 “인체해부학 - 뼈대와 근육"은 일반인과 보건의료 인을 대상으로 인체 구조와 한글 의학 용어에 관하여 수업하는 강좌로, 2017년 9월 25일에서 2018년 1월 14일로 종료되어 과 제 제출, 토론 참여 및 이수증 발급은 안 되었지만 강좌, 텍스트 및 퀴즈는 열람할 수 있어 청강 신청을 하였다. 네 번째로 “내 안 의 우주: 인체 구조와 기능 1 "은 의학적 관점에서 누구나 인체의 구조와 기능을 이해할 수 있도록 하는 강좌로, 2018년 11월 12 일부터 2018년 12월 30일까지로 진행 중이어서 수강 신청을 하 였다. 마지막으로 치료·보건의 "WHY, HOW로 풀어보는 생리 학”은 네 번째 강좌와 동일한 생리학 영역의 강좌로 간호 및 보 건의료인을 대상으로 하며, 2018년 11월 26월부터 2019년 2월 3 일까지로 역시 진행 중이어서 수강 신청을 하였다. 이렇게 총 3 개의 기초 의학 강좌를 최종적으로 선정하여 상호작용 설계전 략을 분석하였다. 또한 2018년 현재 K-MOOC에 개설되어 있 는 치의학 강좌가 없어 해외 치의학 교육 분야의 MOOC 강좌를 추가 조사하였다.

\section{연구 방법과 자료 분석}

본 연구에서는 선정된 해부학과 생리학의 3 개의 강좌를 직접 수강 및 청강 신청한 후, 학습자로서 학습 활동에 참여하면서 강
좌의 구성 요소와 적용된 상호작용 설계전략을 분석하였다. 먼 저 Byun 등[15]의 연구에서 제안한 $\mathrm{K}-\mathrm{MOOC}$ 강좌 시 적용 가 능한 상호작용의 구체적인 설계 지침을 분석의 기준으로 설정 하였다. Byun 등[15]이 제안한 기준은, $\mathrm{K}-\mathrm{MOOC}$ 와 $\mathrm{MOOC}$ 강 좌를 찾아 수강하여 Moore의 세 가지 유형의 상호 작용을 기반 으로 공통적 설계 전략을 도출하여, 수강지속 의도와 학습 효율 성을 높일 수 있는 교수설계 전략 지침이다.

Byun 등[15]이 제시한 상호작용 설계 세부 전략을 토대로, $\mathrm{K}-$ $\mathrm{MOOC}$ 의 기초 의학 강좌에서 교수설계 전략이 어떻게 적용되 었는지를 분석하였다. 분석한 내용과 관련 정보를 분류하고, 연 구자들은 공동으로 검토하고 상호 의견을 수렴 및 협의하여 적 용된 설계 전략 요소와 강좌별 특징을 분석하였다.

이와 더불어 해외 치의학 교육 분야의 $\mathrm{MOOC}$ 개설 여부를 확 인하였다. MOOC의 급격한 양적 성장과 강좌의 다양함으로 인 한 학습자의 혼란을 줄이고 적절한 강좌의 선택을 돕기 위해 다 양한 플랫폼의 강좌 목록을 모아 둔 $\mathrm{MOOC}$ 강좌 목록 사이트 인 Class Central (https://www.classcentral.com/)과 MOOC List (https://www.mooc-list.com/)에서 해당 강좌의 목록을 검색하여 현재 개설되어 운영 중인 치의학 분야 MOOC의 현황 을 살펴보았다.

또한 평생교육의 일환으로 치과의사의 보수교육을 위해 대한 치과의사협회에서 현재 활용하고 있는 온라인 교육도 함께 살 펴보았다. 이러한 연구 결과를 종합하여 향후 치의학 교육에서 $\mathrm{K}-\mathrm{MOOC}$ 의 강좌 개발과 운영을 위한 실제적 방향성을 모색하 고 제시하고자 하였다. 
Table 1. Free online dentistry courses in Massive Open Online Courses

\begin{tabular}{|c|c|c|c|c|c|}
\hline Course Name & Main body of operation & Provider & Cost & Nation & Language \\
\hline $\begin{array}{l}\text { The Oral Cavity: Portal } \\
\text { to Health and Disease }\end{array}$ & University of Pennsylvania & Coursera & Free course auditing & USA & English \\
\hline Materials in Oral Health & The University of Hong Kong & Coursera & Free course auditing & Hong Kong & English \\
\hline $\begin{array}{l}\text { Improving Your Image: } \\
\text { Dental Photography in Practice }\end{array}$ & University of Birmingham & FutureLearn & Free course auditing & UK & English \\
\hline Implant Dentistry & The University of Hong Kong & Coursera & Free course auditing & Hong Kong & English, Chinese \\
\hline Discover Dentistry & The University of Sheffield & FutureLearn & Free course auditing & UK & English \\
\hline Endodoncia Avanzada & Universidad Europea de Madrid & Miriada X & Free & Spain & Spanish \\
\hline $\begin{array}{l}\text { Caries Management by } \\
\text { Risk Assessment }\end{array}$ & $\begin{array}{l}\text { University of California } \\
\text { San Francisco }\end{array}$ & Coursera & Free & USA & English \\
\hline Farmacologia para odontologos & $\begin{array}{l}\text { Universidad Nacional Autónoma } \\
\text { de México }\end{array}$ & Coursera & Free course auditing & Mexico & Spanish \\
\hline $\begin{array}{l}\text { Ortodoncia:tratamientos } \\
\text { dentales sencillos para } \\
\text { casos complejos }\end{array}$ & The Pontificia Universidad Javeriana & edX & Free & Colombia & Spanish \\
\hline $\begin{array}{l}\text { Manejo moderno dela } \\
\text { caries dental }\end{array}$ & $\begin{array}{l}\text { Universidad Nacional Autónoma } \\
\text { de México }\end{array}$ & Coursera & Free course auditing & Mexico & Spanish \\
\hline $\begin{array}{l}\text { Introduction to Dental } \\
\text { Medicine }\end{array}$ & University of Pennsylvania & Coursera & Free course auditing & USA & English \\
\hline
\end{tabular}

\section{결 과}

\section{의학 분야 K-MOOC 강좌의 상호작용 설계전략 분석}

정부 주도로 운영되는 $\mathrm{K}-\mathrm{MOOC}$ 는 사이트에 명시된 추진계 획에 맞춰 2015년 이래 양적·질적으로 급속히 성장하여 국내 유수대학이 개발한 양질의 강좌를 서비스하고 있다. 의약 분야 의 강좌는 2018년 12월 기준 총 30개로 전체 $6 \%$ 에 해당하는 비 율로 운영 중이었고, 치의학 교육에 활용할 수 있는 기초 의학 강좌는 5 개뿐이었다. 이들은 모두 무료로 수강 가능하며 일정 이상의 수업 참여 시간과 일정 학업성취도 기준에 도달하면 이 수증을 발급받을 수 있다.

연구자들이 K-MOOC에서 직접 수강한 "인체해부학 - 뼈대 와 근육", “내 안의 우주: 인체 구조와 기능1”, "WHY, HOW로 풀어보는 생리학"의 3 개의 기초 의학 강좌를 상호작용 요소별 설계전략에 따라 분석한 결과, Appendix 1에 제시한 것처럼 공 통적으로 전통적 1 인 교수자 중심의 강의식 교실 수업을 온라 인으로 옮겨 놓은 $\mathrm{MOOC}$ 의 초기 버전과 유사하였다. 또한 실제 대학 수업에서 함께 연계하여 이루어지고 있는 실험실을 활용 한 실험실습에 관한 내용은 다루어지고 있지 않았다.

그러나 콘텐츠의 구조적 측면의 설계에서는 $\mathrm{MOOC}$ 의 설계 원리에 따라 내용전문가인 교수자, 교수설계자(instructional designer) 및 제작전문가의 협동적 작업으로 설계의 기본 원칙 과 설계전략에 맞추어 잘 이루어져 있었다. 특히 동일 학문을 다
루는 "내 안의 우주: 인체 구조와 기능1"과 "WHY, HOW로 풀 어보는 생리학"은 거의 유사한 콘텐츠의 내용과 순서를 가졌다. 이 밖에도 공통적으로 강좌 소개, 다운로드 가능한 강의 자료의 텍스트 제공 및 강의 영상의 영어 자막 제공으로 효과적으로 학 습 자원이 전달될 수 있도록 하였다. 또한 조교와 운영자를 통한 피드백, 학습 주차별 퀴즈의 응답 기회 제한, 게시판을 통한 토 론 내용의 공유 및 테크놀로지의 활용 등 학습 효과를 높일 수 있는 전략들이 공통적으로 사용되었다. 특히, 컴퓨터 그래픽을 활용한 입체적 영상 및 시각 자료의 제공은 학습자가 효율적으 로 학습할 수 있는 장점으로 들 수 있다(Fig. 1).

Appendix 2에 제시한 것과 같이 일부 강좌의 경우 학습자와 의 상호작용을 유도하여 학습 효율성을 높이기 위한 설계 전략 을 사용하고 있었다. 그러나 의학 학문의 특성상 많은 이론 학습 내용으로 교수자 중심의 일방향 정보 전달 형식이 주를 이루었 으며, 매 주차 강의 동영상 시간도 모두 20 분 정도 분량으로 제 작되어 있었다. 또한 학습 내용 역시 기초적 수준이었으며 객관 식 퀴즈와 간단한 서술형 문제에 대한 답을 요구하는 토론 과제 로 인하여 학문의 전문성 및 학습 효과성에 있어서는 부족한 부 분이 많았다.

\section{치의학 학문의 $\mathrm{MOOC}$ 강좌와 실태}

$\mathrm{K}-\mathrm{MOOC}$ 의 치의학 강좌의 개설이 전혀 없어, 공유와 공개라 는 MOOC의 원칙에 따라 2018년 현재 해외 MOOC에서 치의 
학 교육 관련 무료로 수강할 수 있는 강좌의 개설 여부를 확인한 결과, Table 1 에 제시된 것과 같았다. 총 11 개의 강좌가 최근에 서비스되기 시작하였고, 그중 세 강좌가 무료로 수강할 수 있었 으며 나머지는 청강할 수 있었으나 이수증 발급은 유료로 진행 되었다. 7 개의 강좌가 영어로 진행되었으며, 제공기관 역시 미 국의 $\mathrm{MOOC}$ 사이트인 Coursera가 대부분을 이루고 있었다.

그 외, $\mathrm{MOOC}$ 가 아닌 형태의 온라인 교육이 대한치과의사협 회에서 치과의사의 평생교육 일환으로 보수교육을 위해 활용되 고 있다. 이러한 온라인 교육은 기업의 이러닝과 같이 직업 능 력 향상을 위해 온라인을 통해 일방향의 콘텐츠를 전달하는 8 개 의 강좌가 개설되어 있다. 이들 온라인 교육은 1 인 교수자에 의 해 진행되는 교육용 동영상을 학습자가 온라인을 통하여 단순 히 보고 들으며 학습하는 형태로 학습자와의 상호작용이 거의 고려되어 있지 않았다. 반면 강좌 당 보수교육점수 1점을 부여 받을 수 있었지만, 유료로 운영되었고 첫 수강 시 한 번만 이수 에 반영하였다. 또한 2017년 1월부터 국민건강보험공단에서 의 료인을 대상으로 금연치료 의료인 온라인 교육을 시행하여 운 영하고 있다.

\section{고 찰}

MOOC가 2011년 컴퓨터공학 분야에서 먼저 서비스를 시작 한 이후 빠른 성장과 참여 분야의 확대로, 2013년 기준 건강과 의학 관련 분야에서도 225 개의 강좌가 확인되며 전 분야에서 성장이 있었다[2,7]. 이에 따라 본 연구에서 살펴본 결과 $\mathrm{MOOC}$ 의 치의학 관련 강좌는 2018 년 12 월 현재 총 11 개의 강좌가 개 설되어 있었고, 2013년에 10월 FutureLearn을 통하여 "Discover Dentistry"의 하나의 강좌가 시작되었으며 대부분 최근 에 강좌 운영을 시작하였다[10]. 또한 현재 $\mathrm{K}-\mathrm{MOOC}$ 에서는 치 의학 강좌의 개설이 전혀 없는 실정이다.

이처럼 치의학 교육에서 $\mathrm{MOOC}$ 의 도입이 늦고 강좌 수가 적 은 이유는 치의학 교육이 실험 실습 등 학습자의 직접적 참여 와 실제적 학습 활동을 경험할 수 있는 학습자, 교수자, 환자와 의 직접적 접촉을 중요하게 여겨 온라인 매체를 이용한 교육에 대한 관심이 적기 때문이다[9]. 이와 관련하여 미국치의학교육 협의회(American Dental Education Association) 역시 치과는 환자와의 접촉을 통한 긴 진료 시간을 가지기 때문에 치과 의료 전문성을 기르기 위해서는 직접적 대인관계를 통한 상호작용을 바탕으로 한 교육이 필수적이라고 서술하고 있다[27].

$\mathrm{K}-\mathrm{MOOC}$ 에서 개설되어 있는 기초 의학 강좌는 총 5 개로 다 른 학문과 비교하여 매우 적게 개설이 되어 있었다. 본 연구에 서는 그 중 기초 치의학과 유사하고 수강 및 청강 신청이 가능한 세 강좌를 최종적으로 선정하여 상호작용 설계 전략을 분석하
였다. 그 결과, 이들 세 강좌는 1 인 교수자 위주의 강의실 수업 형태의 강의 스타일, 20 분 정도의 강의 동영상, 동일 교과목 강 좌의 설계 및 운영으로 제공자 중심의 일방향 콘텐츠 전달 형태 로 운영되고 있었다. 그리고 학습 대상을 일반인과 보건의료인 을 동시에 설정하여 양질의 학습 내용이 설계되지 못하였고, 단 순 객관식 형태의 평가 방식 등은 학습자와 교수자 및 다른 학습 자와의 상호작용을 어렵게 하였고, 지식수준의 낮은 학습성과 를 목표로 하고 있었다. 그 밖에 강의 영상 전후에 참여 대학이 나 병원의 환자 유치를 위한 홍보 영상이 불필요하게 많이 내재 되어 있었다. 따라서 향후 치의학 교육에 적용하기 위해서는 먼 저 특정 학습자 집단의 필요와 요구에 따라 차별화된 강좌 설계 가 필요하겠다.

반면, 강좌의 설계에 있어서는 교수자인 내용전문가, 교수설 계자 및 제작 전문가의 협동적 작업으로 설계의 기본 원칙과 전 략에 맞추어 잘 이루어져 있었다. 특히 콘텐츠의 경우 교수자의 음성만으로 이해하기 어려운 부분을 적절한 텍스트의 사용과 3 차원 컴퓨터 그래픽 활용 등으로 입체적으로 잘 표현이 되어 학 습의 이해를 도왔다. 이는 의학과 치의학에서 학습자들이 시각 적 입체 자료를 더 선호하는 점과 한국인의 온라인 시각 문화의 발달과 함께 $\mathrm{K}-\mathrm{MOOC}$ 강좌 설계 시 장점으로 이용될 수 있겠 다[18,21,28].

치의학 교육에서 면대면 접촉을 통한 상호작용의 강조와 $\mathrm{MOOC}$ 의 특성인 불특정 다수의 학습 대상 설계로 양질의 고 등교육의 제공 어려움 때문에, Kearney 등[9]과 Harder [23]는 $\mathrm{MOOC}$ 가 치의학 교육에 완전히 적용되는 것은 시기상조라고 하였다. 그러나 먼저 의학 교육과 마찬가지로 치의학 분야에도 공공 의료, 학생 및 의료인의 평생 교육에 있어 긍정적인 교육 효과가 기대되고[7], 다양한 임상환경에서의 의사결정과 기본적 기술의 환자에 대한 적용을 위한 보다 향상된 방식의 학습이론 이 적용된 교육방식의 적용 요구가 있다. 또한 $\mathrm{MOOC}$ 를 이용하 는 고등교육 수요자의 요구와 이들의 사회·문화적 배경이 다양 하다[1,5,18]. 그 결과 치의학 교육 종사자들 역시 이러한 고등 교육의 변화를 긍정적으로 인식하고 다양한 시도를 통한 연구 를 진행하고 있다[9,10,28]. 따라서 본 연구 결과를 토대로 앞으 로 치의학 교육에서 $\mathrm{K}-\mathrm{MOOC}$ 의 적용을 위한 실제적 방향성을 정리하면 다음과 같다.

향후 적용을 위해 가장 중요한 점은 학습자의 필요와 요구 분 석을 통한 학습 대상의 설정이다. 즉, 치과대학 학생, 치과의사, 그 외 보건의료계통 종사자를 포함한 일반인으로 크게 세 범주 로 나누어 차별화된 강좌를 설계할 필요가 있다. 먼저 학생은 최 신 테크놀로지 사용에 익숙하며 이러한 기기의 사용을 선호하 며[29], 또한 가상현실(virtual reality)의 실습교육장비를 이용 한 임상 전 치과 실습에서도 교육적 효과가 나타났다[30]. 더불 
어 $\mathrm{MOOC}$ 에 있어서 학생들은 특히 시각적 입체 자료의 제공과 교수자 또는 조교와 운영자를 통한 피드백을 $\mathrm{MOOC}$ 의 강점으 로 뽑았다 $[1,21,28]$. 반면 플립 러닝의 형태와 같이 MOOC을 통 한 사전 학습 후 심화 토론의 경우 학습의 효과는 있었으나, 학 생들은 방대한 학습 자료와 많은 학습요구시간을 단점으로 뽑 았다. 따라서 교육 내용과 분량의 표준화를 통한 $\mathrm{K}-\mathrm{MOOC}$ 의 학생 교육에의 적용은 학습 자료의 개발과 관리에 대한 교수자 의 부담을 줄이고 학생들의 학습 효과를 증대시킬 것이다.

다음은 직업 능력 향상을 위해 보수교육이 의무화되어 있는 치과의사를 포함한 의료인을 위한 유료 K-MOOC 강좌 설계 이다. 의무교육이 필수로 지정되어 있는 국내 의료인들은 손쉽 게 강좌를 이수할 수 있으며, 또한 한류의 열풍과 함께 동남아를 비롯한 한국에 우호적인 국가들에게 의료 및 교육 수출을 기대 할 수 있다. 2016년 K-MOOC 콘텐츠 개발 비용은 강좌당 평균 1.1 억으로 이러한 초기 투자비용이 부담으로 작용하고 있다[1]. 이에 교육부와 보건복지부, 대한치과의사협회와 같은 관계기관 및 참여 대학과의 협업을 통한 유료 강좌 개설 및 운영은 이러한 비용 부담을 줄일 수 있다.

마지막으로 $\mathrm{K}-\mathrm{MOOC}$ 에 참여한 일반인의 경우 지적 호기심 과 개인적 목적으로 학습에 참여하였으나, 학습내용의 난이도 또는 선수학습요건의 제한으로 학습을 중도에 포기하는 경우가 많다[1]. 따라서 보건의료계통 종사자를 포함한 일반인을 대상 으로 한 강좌 설계는 주요 건강 주제를 다루면 공공보건 교육의 가능성 및 의사와의 언어적 간격을 극복하여 국민 보건 건강의 향상을 기대할 수 있다.

위에서 제시한 방향을 적용하여 학습자 맞춤형 강좌 개발과 제공이 K-MOOC를 통해 이루어진다면 치의학 교육 분야의 다 층적이고 광범위한 학습자의 요구를 충족시킬 뿐만 아니라, 보 건 의료 분야에서도 K-MOOC의 활발한 이용을 기대할 수 있 다. 또한 최근 $\mathrm{MOOC}$ 에 개설되어 운영 중인 치의학 관련 강좌 의 공통적 설계 전략 및 멀티미디어 활용 디지털 자료의 개발전 략을 추가 연구를 통하여 도출한다면 아직 개발되지 않은 국내 치의학 교육의 $\mathrm{K}-\mathrm{MOOC}$ 강좌 설계에 대한 포괄적인 실천적 지 침을 제공할 수 있을 것이다.

\section{ACKNOWLEDGEMENTS}

This study was supported by a research fund from Chosun University, 2016.

I would like to thank to Professor Heung-Joong Kim of Anatomy and Professor Do Kyung Kim of Physiology who helped to analyze the data as contents experts.

\section{CONFLICTS OF INTEREST}

The authors declare that they have no competing interests.

\section{ORCID}

\author{
Jieun Lee \\ https://orcid.org/0000-0003-2475-6112 \\ Sun-Kyoung Yu \\ https://orcid.org/0000-0003-0801-1663
}

\section{REFERENCES}

1. Kee Y. Global MOOC's learning and analysis of K-MOOC learning experience. J Educ Cult 2018;24:65-85.

2. Lee SJ. Prospect and tasks of MOOC. Educ Res Stud 2013;19:107-139.

3. K-MOOC [Internet]. Seoul: National Institute for Lifelong Education; 2015 Oct [cited 2019 Mar 1]. Available from: http://www.kmooc.kr/.

4. Kim JM, Lee WK, Kim Y, Kim MJ, Ahn YH. Activation plan of online lecture for linking Korean type MOOC. Seoul: Research and Business Foundation Korea University; 2014 Dec. 142p.

5. Choi KA. Tasks for effective use of $\mathrm{K}-\mathrm{MOOC}$ as a higher continuing education. J Lifelong Learn Soc 2016;12:1-24. doi: 10.26857/JLLS.2016.05.12.2.1.

6. Kim SR. '명교수 명강의' 어디서나 무료로 듣는다. Korean Faculty Member Newspaper 2016. [cited 2019 Mar 1]. Available from: http://blog.naver.com/ktcu_attic/220595908393/.

7. Liyanagunawardena TR, Williams SA. Massive open online courses on health and medicine: review. J Med Internet Res 2014;16:e191. doi: 10.2196/jmir.3439.

8. Valachovic R. Is Dental Education Ready for MOOCs? [Internet]. American Dental Education Association; 2013 Jul 15 [cited 2019 Mar 1]. Available from: https://adeachartingprogress.wordpress.com/2013/07/15/is-dental-education-ready-for-moocs/

9. Kearney RC, Premaraj S, Smith BM, Olson GW, Williamson AE, Romanos G. Massive open online courses in dental education: two viewpoints: viewpoint 1: massive open online courses offer transformative technology for dental education and viewpoint 2: massive open online courses are not ready for primetime. J Dent Educ 2016;80:121-127.

10. Stokes CW, Towers AC, Jinks PV, Symington A. Discover dentistry: encouraging wider participation in dentistry using a massive open online course (MOOC). Br Dent J 2015;219:81-85. doi: 10.1038/sj.bdj.2015.559.

11. Nam SE, Cho KL. Interaction patterns in Korea Massive 
Open Online Course (K-MOOC) through a case study. Korean Assoc Educ Inf Media 2017;12:735-762.

12. Suh H. Kang M. Relationships among learner characteristics, peer interactions, and learning outcome in the online learning community. J Educ Technol 2005;21:1-28. doi: 10.17232/KSET.21.2.1.

13. Kim KD, Ko Y, Choi KU, Park IW. A study on effective factors on academic achievement in e-learning environments. Korea Educ Rev 2012;18:169-188.

14. Moore MG. Editorial: three types of interaction. Am J Distance Educ 1989;3:1-7. doi: 10.1080/08923648909526659.

15. Byun M, Lee J, Hong S, Cho H, Cho M. Instructional strategies to promote interaction in K-MOOC: focused on Moore's three types of interaction. J Korean Assoc Educ Inf Media 2016;22:633-659. doi: 10.15833/KAFEIAM.22.3.633.

16. Lee HJ. New perspectives of theoretical researches in webbased distance education: beyond Moore's concepts. Korean J Educ Res 2004;42:137-168.

17. Hew KF. Promoting engagement in online courses: what strategies can we learn from three highly rated MOOCs. Br J Educ Technol 2014;47:320-341. doi: 10.1111/bjet.12235.

18. Jung IS. MOOCs global learning age understanding MOOCs. Seoul: Hakjisa; 2015;47-73.

19. Choi JS. A cultural revolution in online education: MOOC. J Glob Cult Contents 2014;14:179-198.

20. National Institute for Lifelong Education. 'K-MOOC 시 범 운영 7 개월의 성과’: 수강신청 10 만, 방문건수 100 만 돌파. Seoul: National Institute for Lifelong Education; 2016 May 11 [cited 2019 Mar 1]. Available from: http://www.nile.
or.kr/contents/contents.jsp?bkind=basic\&bcode=CADAAA A\&bmode=view\&idx=BCJDFCECEBJEG\&pageNo=1

21. Swinnerton BJ, Morris NP, Hotchkiss S, Pickering JD. The integration of an anatomy massive open online course (MOOC) into a medical anatomy curriculum. Anat Sci Educ 2017;10:53-67. doi: 10.1002/ase.1625.

22. Gooding I, Klaas B, Yager JD, Kanchanaraksa S. Massive open online courses in public health. Front Public Health 2013;1:59. doi: 10.3389/fpubh.2013.00059.

23. Harder B. Are MOOCs the future of medical education? BMJ 2013;346:f2666. doi: 10.1136/bmj.f2666.

24. Hoy MB. MOOCs 101: an introduction to massive open online courses. Med Ref Serv Q 2014;33:85-91. doi: 10.1080/02763869.2014.866490.

25. Tucker B. The flipped classroom: online instruction at home frees class time for learning. Educ Next 2012;12:82-83.

26. Lee SS. Design principles of interactions for blended learning. J Korean Assoc Educ Inf Media 2007;13:225250.

27. ADEA statement on professionalism in dental education: (as approved by the 2009 ADEA House of Delegates). J Dent Educ 2017;81:885-890.

28. Grimes EB. Student perceptions of an online dental terminology course. J Dent Educ 2002;66:100-107.

29. Sin JH, Kim MS, Choi JY, Heo YS, Lee JE. Educational psychology. Seoul: Kyoyookbook; 2015.

30. Jung HS, Kim H, Moon SY. Virtual reality training simulator for tooth preparation techniques. Oral Biol Res 2018;42:235-240. doi: 10.21851/obr.42.04.201812.235. 
Appendix 1. Characteristics of basic medical science courses based on detailed instructional design strategies for interaction in K-MOOC

\begin{tabular}{|c|c|c|c|}
\hline $\begin{array}{l}\text { 상호작용 } \\
\text { 유형 }\end{array}$ & 상호작용 요소 & $\mathrm{K}-\mathrm{MOOC}$ 에서 적용 가능한 상호작용 설계 세부 전략 & $\begin{array}{l}\mathrm{K}-\mathrm{MOOC} \text { 에서의 } \\
\text { 기초 의학 }\end{array}$ \\
\hline \multirow{10}{*}{$\begin{array}{l}\text { 학습자- } \\
\text { 콘텐츠 간 } \\
\text { 상호작용 }\end{array}$} & \multirow[t]{3}{*}{ 텍스트 } & $\begin{array}{l}\text { 1. 본격적인 모듈에 들어가기 전에, 강좌 소개에서 강좌에 대한 충 } \\
\text { 분한 정보를 영상이나 텍스트로 제공하라. }\end{array}$ & 영상이나 텍스트로 제공 \\
\hline & & $\begin{array}{l}\text { 2. 강좌 개발 시 핸드아웃이나 참고자료, 심화 학습내용들을 제공 } \\
\text { 하고, 추가 학습 자료를 제공하라(PDF, 심화 유튜브 영상 링크, } \\
\text { 비디오 script, reference list, simulation tool 등). }\end{array}$ & 강의 자료의 $\mathrm{PDF}$ 제공 \\
\hline & & $\begin{array}{l}\text { 3. 강의 영상이 끝나고 학습 정리 및 추가 토론거리에 대한 내용을 } \\
\text { 텍스트 및 사진자료로 올려 상호작용이 가능하도록 하라. }\end{array}$ & 제공하지 않음 \\
\hline & \multirow[t]{3}{*}{ 교육 영상 } & $\begin{array}{l}\text { 1. 강의자 한 명만 노출되어 강좌를 진행하는 형태에서 벗어나, 주제 } \\
\text { 에 따라 다양한 강의자(혹은 관련 전문가)를 섭외하여 제작하라. }\end{array}$ & 1인 교수자 \\
\hline & & $\begin{array}{l}\text { 2. 한 강좌 안에 다양한 유형의 강의를 구성하라(인터뷰 방식, 다 } \\
\text { 른 전문가들과의 대담 방식 등). }\end{array}$ & 제공하지 않음 \\
\hline & & $\begin{array}{l}\text { 3. 학습자가 자신의 실제 생활에서 강의 콘텐츠와 연계할 수 있는 } \\
\text { 실제적인 문제를 제기하거나, 실생활과 관련된 영상을 제작 삽 } \\
\text { 입하는 툴을 제공하라. }\end{array}$ & 제공하지 않음 \\
\hline & 오디오 영상 & $\begin{array}{l}\text { 1. 단순한 영어 자막 파일을 제공하는 것을 넘어 학습자에게 직접 } \\
\text { 교수자의 육성이 담긴 음성 파일을 팟캐스트 등을 활용하라. }\end{array}$ & 제공하지 않음 \\
\hline & \multirow[t]{2}{*}{ 내재적 내용 } & $\begin{array}{l}\text { 1. 학습자가 배운 내용을 동시적으로 확인하며 수업을 수강할 수 있 } \\
\text { 도록 pop quiz, pre-quiz등 다양한 형태의 수행 과제를 제공하라. }\end{array}$ & 제공하지 않음 \\
\hline & & $\begin{array}{l}\text { 2. 학습자 스스로 배운 것을 직접 생활에 적용해 볼 수 있는 문제를 } \\
\text { 제시하고 해결할 수 있는 기회를 제공하라. }\end{array}$ & 강의 도중 해당 내용 언급 \\
\hline & 교훈적 내용 & $\begin{array}{l}\text { 1. 학습자가 실제로 자신의 실생활과 관련하여, 강좌를 통해 배운 } \\
\text { 것들을 활용해 볼 수 있는 가이드라인을 제공하라. }\end{array}$ & 강의 도중 해당 내용 언급 \\
\hline \multirow{10}{*}{$\begin{array}{l}\text { 학습자- } \\
\text { 교수자 간 } \\
\text { 상호작용 }\end{array}$} & \multirow[t]{2}{*}{ 교수자의 강의 스타일 } & $\begin{array}{l}\text { 1. 교수자의 움직임과 목소리가 단조롭지 않도록 수시로 모니터링 } \\
\text { 하며 제작하고, 비언어적 표현 요소를 가미하라. }\end{array}$ & $\begin{array}{l}\text { 강의실 수업 형식 또는 } \\
\text { EBS 인터넷 강의 형식 }\end{array}$ \\
\hline & & $\begin{array}{l}\text { 2. 중간 중간에 다른 사람과의 인터뷰, 짧은 관련 동영상을 첨부하 } \\
\text { 여 반복되어 노출되는 강좌에 대한 지루함을 최소화하라. }\end{array}$ & 제공하지 않음 \\
\hline & 비디오 스타일 & $\begin{array}{l}\text { 1. 컴퓨터 그래픽을 활용하여, 학습자가 과목 자체에 갖는 거부감 } \\
\text { 을 해소시키며, 학습 몰입의 방해요소를 줄일 수 있도록 하라. } \\
\text { 2. 강좌 시작 전, 소개에 짧은 애니메이션, 비디오 영상 등을 추가 } \\
\text { 하여 학습자의 흥미를 유발하라. }\end{array}$ & $\begin{array}{l}\text { 3D 영상 등 학습자가 입체적으 } \\
\text { 로 이해할 수 있기 쉽게 제공 } \\
\text { 제공하지 않음 }\end{array}$ \\
\hline & 강좌 시간 & $\begin{array}{l}\text { 1. 강좌 영상은 대체로 } 6 \text { 분에서 } 10 \text { 분 사이를 넘지 않도록 하라 } \\
\text { (6분 미만을 권장). }\end{array}$ & $\begin{array}{l}20 \text { 분정도의 강의 영상 } \\
\text { 총 동영상 시간과 학습인정 } \\
\text { 시간이 매우 긺 }\end{array}$ \\
\hline & \multirow[t]{2}{*}{ 교수자의 권위 } & $\begin{array}{l}\text { 1. 강좌 중간 중간에 학습자가 자신이 배운 내용을 중간에 점검 받 } \\
\text { 을 수 있는 피드백 기회를 제공하라. }\end{array}$ & $\begin{array}{l}\text { 강의 도중 질문 형태로 } \\
\text { 피드백 제시 }\end{array}$ \\
\hline & & $\begin{array}{l}\text { 2. 학습자가 학습을 시작, 종료 시 학습 내용에 대한 정리 이외에 } \\
\text { 학습을 종료한 것에 대한 축하 메시지 영상을 삽입하라. 특히 플 } \\
\text { 랫폼의 단체 메일이나 문자 자동발송 기능을 활용하여 설계하 } \\
\text { 라. }\end{array}$ & 제공하지 않음 \\
\hline & \multirow[t]{4}{*}{ 조교 } & $\begin{array}{l}\text { 1. 강좌 운영 전반에 관한 제반 사항에 대한 즉각적인 피드백 및 공 } \\
\text { 지를 수시로 업데이트할 수 있도록 하라. }\end{array}$ & $\begin{array}{l}\text { 해당 전공의 조교가 } \\
\text { 제공하고 있음 }\end{array}$ \\
\hline & & $\begin{array}{l}\text { 2. 필요한 경우 자동 메일 및 문자 발송시스템으로 조교의 역할을 } \\
\text { 대신할 수 있는 시스템을 설계하라. }\end{array}$ & 제공하지 않음 \\
\hline & & $\begin{array}{l}\text { 3. 단순한 전체 메일을 공지하는 역할을 넘어 교수자가 학습자의 } \\
\text { 과제 해결 및 질의응답 측면에 있어 조력자로서의 역할을 다할 } \\
\text { 수 있도록 설계하라. }\end{array}$ & 제공하지 않음 \\
\hline & & $\begin{array}{l}\text { 4. 정해진 조교 이외에 강좌의 일정 부분까지 이수한 선행 학습자 } \\
\text { 들의 등급을 달리하여 멘토로서 역할을 할 수 있게 설계하라. }\end{array}$ & 제공하지 않음 \\
\hline
\end{tabular}


Appendix 1. Continued

\begin{tabular}{|c|c|c|c|}
\hline $\begin{array}{l}\text { 상호작용 } \\
\text { 유형 }\end{array}$ & 상호작용 요소 & $\mathrm{K}-\mathrm{MOOC}$ 에서 적용 가능한 상호작용 설계 세부 전략 & $\begin{array}{l}\mathrm{K}-\mathrm{MOOC} \text { 에서의 } \\
\text { 기초 의학 }\end{array}$ \\
\hline \multirow{12}{*}{$\begin{array}{l}\text { 학습자- } \\
\text { 학습자 간 } \\
\text { 상호작용 }\end{array}$} & \multirow[t]{3}{*}{ 교수자 평가 } & $\begin{array}{l}\text { 1. 학습 모듈마다 콘텐츠의 특성에 맞는 다양한 평가방식을 설계하 } \\
\text { 라(단계별 평가, 평가+토론 형식 등). }\end{array}$ & $\begin{array}{l}\text { 퀴즈와 중간, 기말의 객관식으로 } \\
\text { 간단한 형식 및 토론이 있으나 } \\
\text { 간단한 서술형의 형식 }\end{array}$ \\
\hline & & $\begin{array}{l}\text { 2. 퀴즈의 경우, 학습자가 지속적으로 오답을 체크하였을 때 일종 } \\
\text { 의 패널티가 가해질 수 있도록 설계하라. }\end{array}$ & 응답기회의 제한 \\
\hline & & 3. 학습자의 수를 예상하여 교수자의 직접 평가를 설계하라. & 제공하지 않음 \\
\hline & \multirow[t]{3}{*}{ 강좌 속 존재감 } & $\begin{array}{l}\text { 1. 강좌를 듣는 학습자 간의 친밀도 및 존재감을 강화하기 위해, 토 } \\
\text { 론 게시판 및 개인 친목교류 게시판은 따로 분류하여 생성토록 } \\
\text { 하라. }\end{array}$ & 제공하지 않음 \\
\hline & & $\begin{array}{l}\text { 2. Ongoing course의 경우 교수자가 이전의 학습 모듈에 있어 학습 } \\
\text { 자들이 제출한 과제물들을 검토한 뒤, 다음 강좌 차시에 직접 학 } \\
\text { 습자의 결과 과제물들을 소개하고 피드백을 줄 수 있도록 하라. }\end{array}$ & 제공하지 않음 \\
\hline & & $\begin{array}{l}\text { 3. 온라인 토론 및 대화를 넘어 학습자 간에 오프라인 미팅을 생성 } \\
\text { 하고 운영해 나갈 수 있는 기회를 제공한다. }\end{array}$ & 제공하지 않음 \\
\hline & \multirow[t]{2}{*}{ 토론 } & $\begin{array}{l}\text { 1. 명목상의 온라인 토론을 생성하여 학습자의 자발적인 참여를 유 } \\
\text { 도하고, 가능한 경우 교수자가 직접 토론에 대한 세부적인 지침 } \\
\text { 과 예시자료를 업로드하여, 학습자 간의 토론이 활성화될 수 있 } \\
\text { 도록 하라. }\end{array}$ & $\begin{array}{l}\text { 토론이 있으나 서술형 문제에 } \\
\text { 대한 답으로, 학습자 간의 } \\
\text { 상호작용보다는 답안만 참고 }\end{array}$ \\
\hline & & $\begin{array}{l}\text { 2. MOOC 안에서의 토론을 넘어, 학습자가 다양한 채에서 소통할 } \\
\text { 수 있도록 Facebook등의 SNS 활용을 연동하라. }\end{array}$ & 제공하지 않음 \\
\hline & \multirow[t]{2}{*}{ 산출물 } & $\begin{array}{l}\text { 1. 학습자가 제출한 과제 게시물의 경우, 게시를 한 뒤에 평가를 받 } \\
\text { 는 전통적인 방식을 벗어나 다른 학습자들 또한 그 게시물을 직 } \\
\text { 접 보고 평가하며 공유할 수 있는 기회를 제공하라. }\end{array}$ & $\begin{array}{l}\text { 제공하지 않음, } \\
\text { 다만 게시물의 공유만 가능 }\end{array}$ \\
\hline & & $\begin{array}{l}\text { 2. 산출물을 제출한 뒤 학습자 간의 피드백을 의무적으로 부여하고 } \\
\text { 학습자의 피드백을 토대로 보완한 2차 산출물을 제출하게 유도 } \\
\text { 하는 등 학습자 간의 교류를 통해 완성해 갈 수 있는 동료를 활용 } \\
\text { 한 학습 기회를 제공하라. }\end{array}$ & 제공하지 않음 \\
\hline & \multirow[t]{2}{*}{ 동료 평가 } & $\begin{array}{l}\text { 1. 학습자가 자신이 제출한 과제물을 다른 학습자와 공유하며 동료 } \\
\text { 평가 및 피드백으로 상호작용할 수 있도록 유도하라. }\end{array}$ & 제공하지 않음 \\
\hline & & $\begin{array}{l}\text { 2. 공유된 타 학습자의 산출물, 저널에 대한 펠로우(follow) 기능 및 } \\
\text { '좋아요 아이콘' 기능 추가로 학습자의 동기 부여 및 친밀감 형 } \\
\text { 성을 유도하라. }\end{array}$ & $\begin{array}{l}\text { 기능은 제공되고 있으나, } \\
\text { 이용은 되고 있지 않음 }\end{array}$ \\
\hline
\end{tabular}

K-MOOC, Korea-Massive Open Online Courses; EBS, educational broadcasting system;3D, 3-Dimensional.

This table analyzed characteristics of basic medical science courses in K-MOOC based on detailed instructional design strategies for interaction proposed by the article of Byun et al. (J Korean Assoc Edue Inf Media 2016;22:633-659) [15]. 
Appendix 2. Specific examples of basic medical science courses in K-MOOC

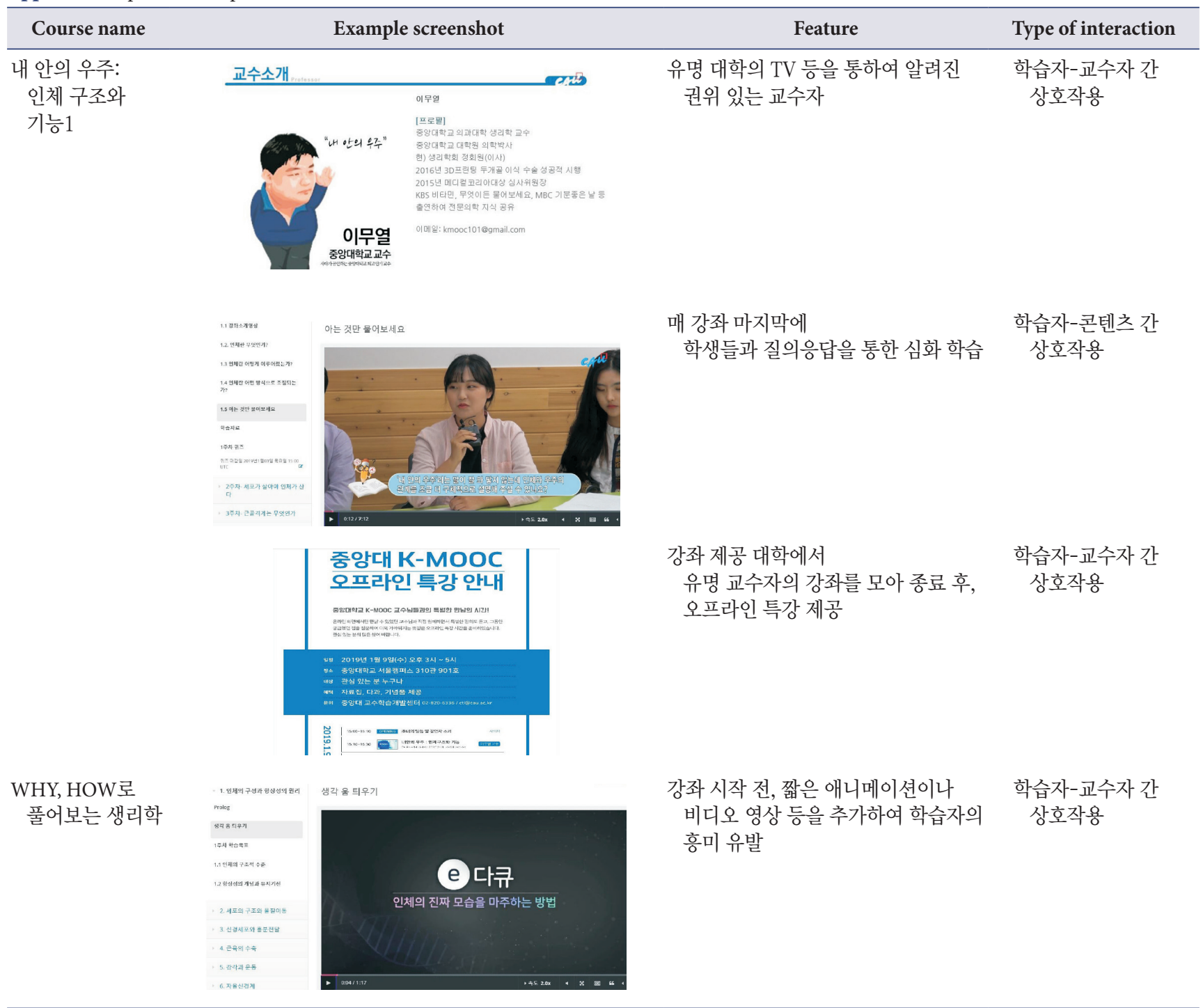

K-MOOC, Korea-Massive Open Online Courses. 